\title{
8. Envolvimento do paciente: desafios, estratégias e limites
}

Margarida Custódio dos Santos e Ana Monteiro Grilo

Neste capítulo, será abordada a temática do envolvimento do paciente no âmbito da segurança. Ao contrário do que ocorre com outras áreas relacionadas a esse tipo de envolvimento, como a adesão ou a satisfação, o papel do paciente nas questões de segurança tem sido ainda pouco estudado e, apesar de resultados positivos de muitas das ações já implementadas, esse é um assunto que necessita ser refletido e incluído na agenda da investigação/pesquisa científica.

Pretendendo abordar as questões principais do envolvimento do paciente em matérias de segurança, o capítulo integra, após a introdução ao tema, um ponto sobre a perspetiva do paciente, referindo-se aí às áreas e tarefas de envolvimento, os determinantes e os limites do envolvimento; o segundo ponto sobre a perspetiva do profissional de saúde, quando se fala sobre o modelo subjacente ao envolvimento do paciente, os desafios, benefícios e limitações dos profissionais; e o terceiro ponto que se centra nas estratégias de promoção do envolvimento.

\section{Participação e envolvimento do paciente nas questões de saúde e doença}

\footnotetext{
"Quem estuda medicina sem livros embarca num mar sem cartas de navegação. Quem estuda medicina sem os pacientes não deve, sequer, embarcar" (Osler 1937).
}

Nos anos 1970, a Organização Mundial da Saúde referia a participação do paciente como um "objetivo desejável das políticas de saúde" e ape- 
lava para a necessidade de implementação de meios de promoção dessa participação (WHO 1978).

Hoje, é consensual que a promoção da participação e do envolvimento do paciente e da família, nos mais variados níveis dos cuidados de saúde, representa benefícios para os pacientes, para os profissionais de saúde e as organizações. De facto, um conjunto alargado de investigações/pesquisas tem demonstrado que o envolvimento do paciente e da sua família está positivamente associado à melhoria dos cuidados de saúde, expressa pelo aumento da satisfação do paciente e da família (Rao, Weinberger, Kroenke 2000), por níveis mais elevados de motivação do paciente, adesão ao tratamento (WHO 2008), menor tempo de recuperação (Adams, Smith, Ruffin 2001) e melhoria da saúde geral do paciente (TNS Qual+ Eurobarometer, 2012).

O envolvimento do paciente tem vindo ainda a ser reconhecido como factor de redução dos custos de saúde e de maior eficiência nos cuidados, quer na fase de diagnóstico, quer no tratamento (Hibbard, Green 2013). Constituindo um determinante importante da relação de colaboração entre o profissional de saúde e os pacientes e/ou seus familiares, o envolvimento do paciente está ainda relacionado com maior transparência na comunicação e menor número de litígios por má prática médica (Studdert et al. 2006).

Efetivamente, tem-se vindo a assistir, nos últimos 40 anos, a uma mudança importante na forma como profissionais de saúde e leigos compreendem o papel do paciente no seu processo de doença/saúde. Essa mudança é determinada, por um lado, pelo aumento da exigência, dos pacientes e das famílias em relação ao conhecimento das questões do diagnóstico e do tratamento e, por outro lado, pelo abandono de uma atitude rigidamente paternalista do médico, que limitava a participação do paciente, negligenciando-a e desincentivando-a.

Nos nossos dias, atitudes mais congruentes com os modelos holista e de centração no paciente têm vindo, progressivamente, a valorizar a participação do paciente, a quem é atribuído um papel ativo na promoção da saúde e na prevenção e tratamento da doença.

A constatação dos benefícios do envolvimento do paciente e da família tem resultado em ações de informação e de educação dos pacientes, familiares e profissionais de saúde (AHRQ 2004; Joint Commission 2004), em recomendações institucionais e normas no âmbito de políticas de saúde (Finkelstein et al 2012; NHS Plan 2000) ou em programas e projetos de inclusão do paciente em questões que lhe dizem respeito (Anvisa 2012; WHO 2006). 
O alargado consenso quanto à importância do envolvimento do paciente não exclui reservas que merecem ser consideradas e serão refletidas neste capítulo.

\section{Envolvimento do paciente nas questões de segurança relacionadas com a saúde e a doença}

Ao contrário do que ocorre em áreas como a adesão ao tratamento ou a decisão de procedimentos médicos, o envolvimento do paciente nas questões de segurança e de prevenção de eventos adversos tem sido menos estudado.

Na discussão sobre o envolvimento do paciente em matéria de segurança, distinguem-se três grandes áreas:

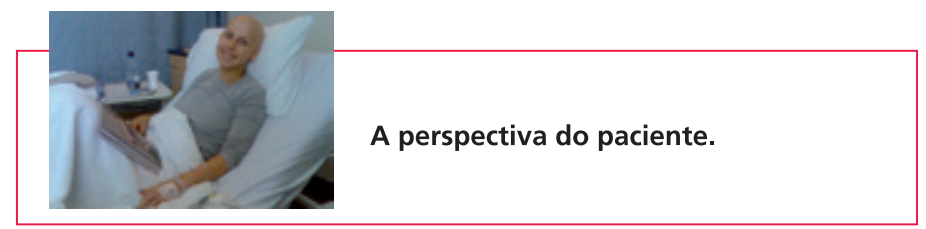

Fonte paciente:http://commons.wikimedia.org/wiki/File:Cancer_patient.jpg - Autor: jennifrog/2008

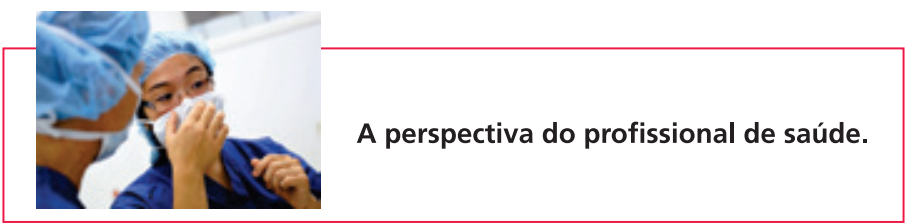

Fonte médicos:http://commons.wikimedia.org/wiki/File:US_Navy_110727-N-YR391-005_Lt._Cmdr._ Jennifer_Freeman,_an_Obstetrics-Gynecology_physician_at_Naval_Hospital_Jacksonville.jpg

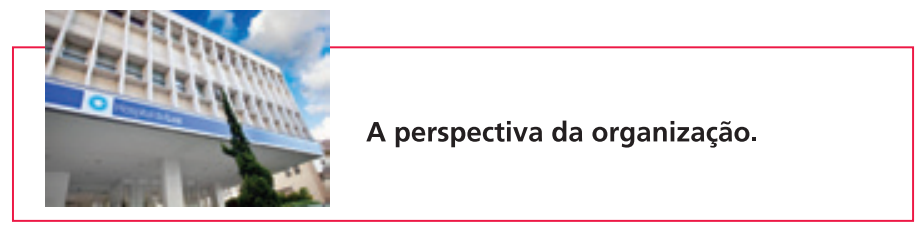

Fonte hospital:http://pt.wikipedia.org/wiki/Ficheiro:Hosp_da_Luz_-_S\%C3\%A3o_Paulo.JPG Autor: Rede Assistencial SP

\section{Envolvimento do paciente na segurança dos cuidados de saúde - perspetiva do paciente}

\section{Áreas e tarefas de envolvimento}

Quando se pensa no envolvimento do paciente nos cuidados de saúde e, especificamente, em matérias de segurança, consideram-se inter- 


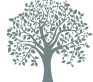

Para saber mais sobre essa questão, veja European Commission (2012). Visite o site: http://ec.europa.eu/index pt.htm rogações como: "O que se espera que o paciente faça?" ou "Qual o grau de fiabilidade/confiabilidade da contribuição do paciente?" e, evidentemente, "Quais os limites que devem ser considerados?" (Por exemplo, "O que ocorre quando o paciente expressa o desejo de não se envolver?" ou "o que se espera de um paciente que, emocionalmente perturbado, não tem condições para esse envolvimento?")

Relativamente ao "que se espera do paciente", vem sendo consensual que seu envolvimento não pode ser percebido como uma panaceia, nem ser regido por uma orientação em que são utilizados protocolos muito rígidos e, como consequência, espera-se o mesmo de todos os pacientes. A constatação da diversidade de respostas dos pacientes às solicitações para o envolvimento tem despoletado/desencadeado investigações/pesquisas, com resultados frequentemente inconsistentes, que apontam ações e níveis de participação muito diversos. A problemática estende-se à própria conceitualização de "envolvimento" e à diferenciação entre as representações de pacientes e de profissionais de saúde, à distinção entre envolvimento e participação, ou à modelização do envolvimento considerando dimensões como a vontade do paciente, as características da doença e o efetivo grau de participação.

Apesar de ser consensual que a discussão e a investigação/pesquisa sobre o papel do paciente em questões de segurança deram apenas os primeiros passos, Vincent e Coultel, num artigo pioneiro publicado em 2002 com o título Patient safety: what about the patient?, além de defenderem que os pacientes desempenham um papel importante na promoção da sua segurança, indicavam, já, possíveis contribuições específicas do paciente em áreas como o estabelecimento do diagnóstico, a monitorização do tratamento e o relato de acidentes adversos (Coluna A do Quadro 1). Mais recentemente, o reconhecimento de que o paciente pode ser um parceiro na discussão da qualidade dos serviços de saúde estendeu sua participação a contextos mais abrangentes (Coluna B do Quadro 1) que vão para além de ações que respeitam, de forma mais restrita, sua segurança.

Quadro 1 - Papel do paciente na promoção da segurança

\begin{tabular}{|l|l|}
\hline Coluna A & Coluna B \\
\hline $\begin{array}{l}\text { Contribuição para o estabelecimento do } \\
\text { diagnóstico. }\end{array}$ & $\begin{array}{l}\text { Monitorização e gestão de tratamentos e } \\
\text { procedimentos. }\end{array}$ \\
\hline Escolha da equipa de saúde mais adequada. & Redução de taxas de infeção hospitalar. \\
\hline $\begin{array}{l}\text { Participação na decisão de tratamento e na } \\
\text { gestão do plano de cuidados. }\end{array}$ & $\begin{array}{l}\text { Desenvolvimento de ações promotoras de } \\
\text { segurança e de qualidade dos serviços. }\end{array}$ \\
\hline
\end{tabular}


Quadro 1 - Papel do paciente na promoção da segurança (cont.)

\begin{tabular}{|l|l|}
\hline Coluna A & Coluna B \\
\hline $\begin{array}{l}\text { Verificação de que o tratamento é devidamente } \\
\text { administrado, monitorizado e seguido. }\end{array}$ & \\
\hline $\begin{array}{l}\text { Identificação e relato de complicações de } \\
\text { tratamento e de eventos adversos e incentivo } \\
\text { de ações de alteração ou remediação. }\end{array}$ & \\
\hline
\end{tabular}

Ao considerarmos as contribuições do paciente indicadas no Quadro 1, é possível identificar quatro áreas principais de envolvimento:

路 Tomadas de decisão em questões médicas de diagnóstico e tratamento.

膡 Monitorização dos procedimentos do diagnóstico e do tratamento.

滕 Identificação e relato de complicações e de eventos adversos.

噒 Participação em ações abrangentes de segurança do paciente no âmbito institucional (retornaremos a esse assunto mais adiante).

\section{Envolvimento nas tomadas de decisão em questões médicas de diagnóstico e tratamento}

A saúde é, por excelência, uma área de tomadas de decisão. E se, no âmbito da saúde, considerarmos as situações de doença, identificaremos, com facilidade, vários processos de tomada de decisão:

噄 a decisão de procura de cuidados médicos;

a decisão do diagnóstico (que inclui todas as decisões de procedimentos de pesquisa e o estabelecimento final da patologia);

踪 a decisão de aceitação do diagnóstico;

滕 a decisão do tratamento e de aceitação do tratamento, não menos importante (senão, a mais importante);

繗 a decisão de implementação (isto é, adesão) ao tratamento.

Os processos de tomada de decisão apresentam, sempre, algum grau de complexidade e, evidentemente, a possibilidade de erro. Iremos considerar, nesse ponto, o envolvimento do paciente nas questões de segurança relacionadas com a prevenção (ou minoração) do erro nas decisões médicas referentes ao diagnóstico, e a prevenção do erro do paciente relacionado com a inadequada adesão ao tratamento. 
Os erros do diagnóstico são frequentes (Zwaan et al. 2010) e põem potencialmente em causa a segurança do paciente. Para além disso, constituem mais de $30 \%$ dos processos por má prática médica (Studdert et al. 2006). No entanto, a inadequada adesão ao tratamento põe potencialmente em risco a evolução positiva da doença e, em consequência, o bem-estar e a segurança do paciente. Interessa, então, saber de que forma a participação do paciente é capaz de contribuir para melhores diagnósticos e maior e mais responsável adesão às recomendações do tratamento.

O envolvimento do paciente nas tomadas de decisão acerca de sua saúde está consagrado em diretivas legislativas (por exemplo, a Diretiva 2011/24/EU Parlamento Europeu; a Politica Nacional de Atenção Básica (2005), emanada pelo Ministério da Saúde, Brasília), e diz respeito à participação ativa do paciente na determinação do diagnóstico, nas decisões do tratamento (por exemplo, na escolha de planos terapêuticos), na escolha de equipas de saúde que mais se adéquem a si e em quem ele confie a sua segurança.

"Ouça o paciente, ele está a dizer-lhe o diagnóstico" (Osler 1937).

A frase, proferida por William Osler, considerado o pai da medicina moderna, tem tanta pertinência hoje como nos anos 1920 do século XX. A lição do professor Osler parece não ter ainda sido completamente aprendida, e os pacientes continuam a dizer que são pouco ouvidos, e suas preocupações pouco consideradas para o diagnóstico. Contudo, a contribuição do paciente, como "especialista sobre si", para o estabelecimento do diagnóstico, não só atempado/oportuno, mas também mais preciso, tem sido confirmada em investigações/pesquisas recentes (Santos et al. 2010; Piker Institut 2010).

Para a participação efetiva do paciente na determinação do diagnóstico, é essencial a valorização de todas as suas queixas, da sua história clínica, das suas crenças em relação a sintomas e do seu conhecimento em relação a si (Joyce-Moniz, Barros 2005). A participação do paciente ainda poderá ser maximizada por sua motivação, pelo papel que ele próprio se atribui na sua relação com os profissionais de saúde e pelo grau de literacia em relação a questões de saúde (Coulter 2011).

Quanto aos benefícios da participação nas decisões do tratamento, apesar do número reduzido de estudos que demonstram evidência científica, não é difícil entender que atitudes orientadas para a par- 
tilha com o paciente são mais facilitadoras do estabelecimento de comunicação entre o profissional de saúde e o paciente (e/ou família), e a comunicação é uma condição indispensável para a segurança do paciente (Santos et al. 2010).

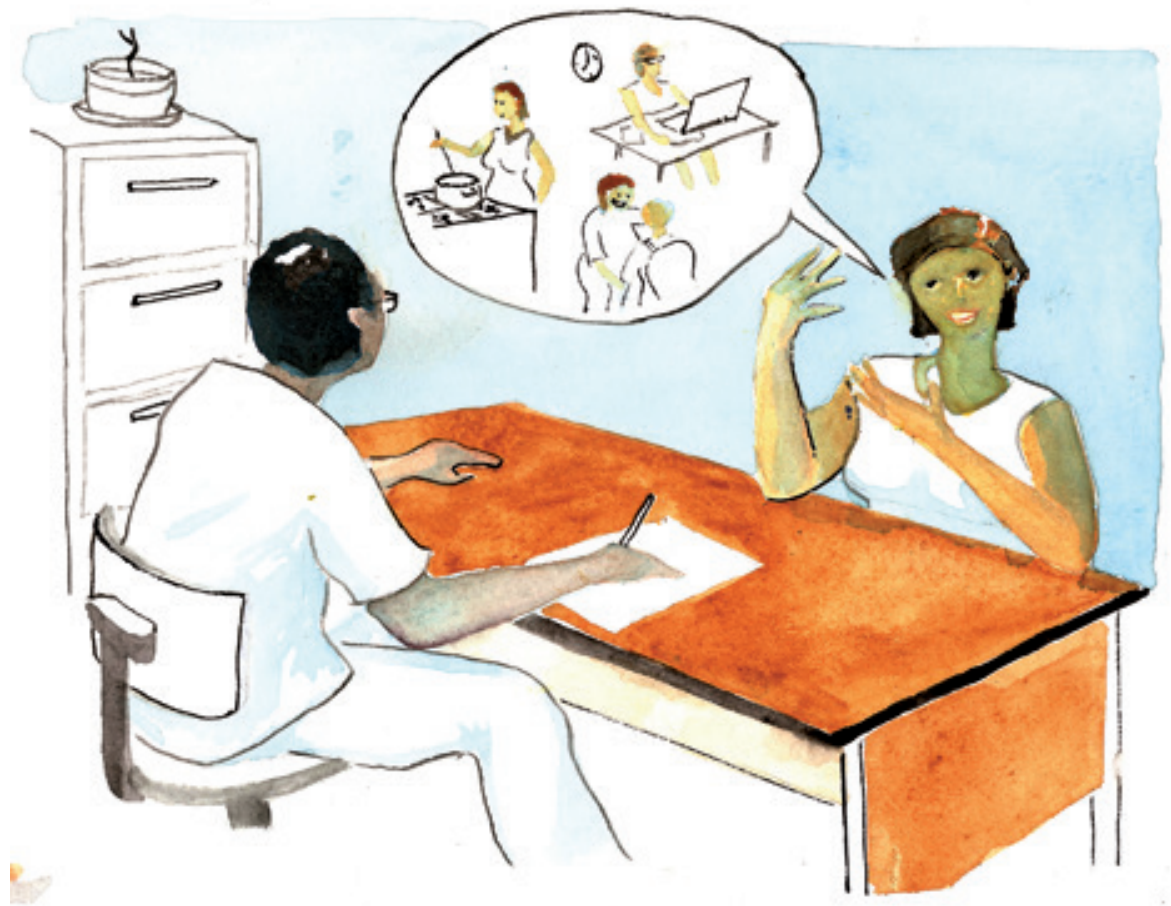

Na realidade, a comunicação será essencial para: a compreensão das preferências do paciente (mesmo em relação ao grau de envolvimento); capacitá-lo quanto à sua doença e às recomendações do tratamento; adequar as decisões ao paciente e às suas competências. O paciente que conhece o tratamento, que percebe e discutiu suas implicações, seus possíveis efeitos secundários e sua efetiva ação na doença estará, com certeza, mais preparado não só para o seguir e não o abandonar em razão de, por exemplo, falsas expetativas, mas também para identificar complicações, atempadamente/oportunamente reportá-las ao médico e com ele discutir as alterações necessárias.

Para além disso, alguns estudos verificaram que a participação do paciente nas decisões de tratamento reduz a prescrição de medicamentos, diminuindo assim o potencial de dano do tratamento (Dentzer 2013), e aumenta a responsabilização do paciente pelo seu tratamento (Coulter, Parsons, Askham 2008). Ouvir o paciente e envolvê-lo nas tomadas de decisão são atitudes que vêm sendo também associadas 


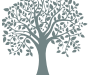

Para saber mais sobre o programa, consulte em National Patient Safety Agency. Clean your hands campaign. Patient empowerment pilot. http://www.thensmc.com/ node/537/pv

Sobre adesão à lavagem de mãos consulte, Measuring HandHygiene Adherence: Overcoming the Challenges (2009). http:// www. jointcommission.org/ assets/1/18/hh_monograph,pdf a níveis mais elevados de adesão ao tratamento e à satisfação com os cuidados (Weingart et al. 2011).

Acresce ainda que, comparativamente a outros tipos de envolvimento na área da segurança, estudos referem essa forma de participação como da preferência de maior número de pacientes, porque é sentida como "menos intrusiva" do que outro tipo de ações, por exemplo, questionar os profissionais sobre a lavagem das mãos ou a esterilização de instrumentos (Waterman et al. 2006).

\section{Envolvimento na monitorização dos procedimentos do diagnóstico e do tratamento}

Nesta área, consideramos importante distinguir entre ações:

\section{Ações de envolvimento do paciente}

Em que o paciente intervém verificando de forma direta as condições de segurança ligadas aos procedimentos a que é sujeito, e outras de automonitorização do tratamento e da evolução clínica.

\section{Quanto à participação do paciente na verificação e monitorização das condições de segurança}

O programa Partners in your care é um exemplo da promoção do envolvimento do paciente num problema antigo, que tem sido provado de difícil resolução: a lavagem das mãos (isto é, os profissionais lavarem as mãos antes e depois de qualquer procedimento que envolva contacto com risco de contaminação). Segundo a Joint Commission on Accreditation on Healthcare Organization (2005), apesar das diversas campanhas, os profissionais de saúde lavam as mãos apenas em 50\% das vezes consideradas adequadas. Com o referido programa, pretendeu-se envolver o paciente nesse cuidado. Os pacientes foram esclarecidos quanto aos objetivos, foi-lhes entregue a brochura "Did You Wash Your Hands?". Os resultados da implementação do programa num Serviço de Cardiologia do Ingham Medical Center, no Michigan, foram considerados positivos, revelaram o aumento do número de lavagens e a diminuição dos índices de infeção hospitalar (Joint Commission Resources 2005; 2006). 
Exemplos semelhantes encontram-se em ações de incentivo do paciente e da família noutras áreas de cuidados, como cirurgias, em que é pedido ao paciente para, na sala de antecâmara da cirurgia, confirmar com o médico o campo cirúrgico (por exemplo, qual é a perna que será submetida à intervenção), o estabelecimento do tratamento, em que é pedida a ação direta do paciente na confirmação da adequação do tratamento (por exemplo, se foram tidos em consideração informações sobre dados de saúde como alergias), ou dos procedimentos do tratamento (por exemplo, confirmando o seu nome no protocolo de tratamento).

O envolvimento do paciente nessas ações tem levantado discussão e suscitado dúvidas que se prendem sobretudo com o acréscimo de responsabilidade do paciente em matérias que não domina (autores falam mesmo na passagem da responsabilidade do médico para o paciente) e com a falta de evidência científica em relação à efetiva eficácia dessas ações na segurança do paciente.

\section{Quanto à automonitorização}

Como já foi referido, os pacientes que são devidamente informados sobre o plano de tratamento e os possíveis efeitos secundários estarão em melhor situação para participar quer no seu autocuidado, quer na gestão do tratamento e na avaliação da evolução clínica. Os benefícios da automonitorização do tratamento têm sido estudados em áreas diversas, desde os tratamentos em patologias cardíacas/de foro cardíaco (Douketis 2001) até os cuidados primários. Como conclusão desses estudos, temos que o paciente pode ser um parceiro relevante na identificação de sinais de complicações do tratamento e, consequentemente, na necessidade e no reajuste dos planos de intervenção, bem como na prevenção de eventos adversos. Um dos estudos de referência nessa área é o realizado por Connock e colaboradores (2007) com o objetivo de analisar a efetividade e custo-benefício de autogestão no tratamento com anticoagulantes orais em comparação com a monotorização de base clínica (realizada por profissionais de saúde). Os autores concluíram que, entre os 16 (trails) estudados, a automonitorização do tratamento tinha melhores resultados que a monitorização clínica mais comum nesses casos, e os resultados foram tão bons como nas situações em que a monitorização clínica era avaliada como de qualidade e efetuada por especialistas.

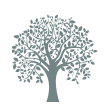

Para o melhor entendimento sobre a discussão acerca desse tema, sugerimos como leitura complementar: Hall et al. (2010), descrito nas referências deste capítulo.

Para um aprofundamento sobre os benefícios da automonitorização, sugerimos a leitura do artigo de Martin, Larsen (2012), descrito nas referências deste capítulo. 


\section{Envolvimento na identificação e relato de complicações e de eventos adversos}

A identificação e o relato de eventos adversos têm sido dois dos focos de maior atenção das ações para a segurança do paciente. Na realidade, se nem sempre os eventos adversos podem ser prevenidos, então eles devem ser cuidadosamente identificados e reportados de forma a reduzir o dano e aumentar o conhecimento de situações de risco. Esses procedimentos podem mesmo ajudar a implementação de processos que levem à sua diminuição. Em conformidade com essa constatação, tem vindo a ser implementados, em muitos países e organizações de saúde, sistemas de análise e reporte de incidentes.

As contribuições dos pacientes para o relato de incidentes adversos é hoje, igualmente, objeto de atenção e investigação/pesquisa, e, de novo, resultados de estudos empíricos revelam alguma inconsistência. No entanto, a prática clínica e alguns programas, implementados quer em contexto hospitalar, quer em ambulatório (por exemplo, em relação a medicamentos), têm revelado benefícios.

A esse respeito, em 2008, Weissmane e colaboradores publicaram um estudo financiado pela Agency for Healthcare Research and Quality em que compararam as respostas de pacientes $(\mathrm{n}=998$ pacientes cirúrgicos pós alta hospitalar) e profissionais de saúde a um questionário sobre eventos adversos ocorridos durante o internamento/internação. Os autores concluíram que os pacientes não só demonstravam ter consciência de eventos que não foram reportados, como, comparativamente, indicavam a ocorrência de um número maior desses eventos. Apesar de, como referido pelos autores, esses resultados não poderem ser considerados "gold standard", e, apesar de questões como a possível confusão por parte dos pacientes entre eventos adversos e situações de diminuída qualidade de prestação, dever ser considerado, parece, neste estudo, claro, que os pacientes internados estão despertos para as questões de segurança e podem ser parceiros na identificação de situações de risco.

\section{Para refletir}

Como você percebe, em sua organização, o nível de envolvimento dos pacientes em questões como diagnósticos, tratamento e segurança do paciente?

Existe algum tipo de incentivo por parte dos profissionais de saúde para maior envolvimento dos pacientes e seus familiares?

Qual é o seu grau de acordo com essa orientação? 


\section{Determinantes e limites do envolvimento}

Após serem referidas as áreas de envolvimento, cabe, agora, mencionar a questão relativa aos determinantes e aos limites desse envolvimento. Partimos para essa discussão tendo como referência os resultados dos estudos nessa área, segundo os quais não só o grau de motivação para o envolvimento é muito diverso, como também os pacientes parecem mais disponíveis a se envolver em determinadas áreas e tarefas em detrimento de outras.

Em relação aos determinantes para o envolvimento, são sobretudo referidos factores relacionados com:

a) o próprio paciente;

b) a doença e as tarefas; e

c) os profissionais de saúde.

\section{Quanto aos determinantes do próprio paciente}

O envolvimento tem provado estar associado a características demográficas, particularmente a idade, o género, os níveis de educação (em especial de literacia médica) e as redes de apoio. Assim, encontram-se níveis mais elevados de envolvimento em indivíduos mais jovens; nas mulheres, com níveis mais elevados de literacia e com redes de apoio familiar (nesses casos, considerando também o envolvimento da família). Em relação a esses resultados, não é difícil entender que a idade e a literacia estão também a elas associadas. Na realidade, na grande maioria das sociedades de hoje, assiste-se ao aumento da literacia das camadas mais jovens e, consequentemente, a níveis mais elevados de sentimento de autoeficácia e de motivação para a participação do indivíduo nas mais variadas áreas de vida.

Figura 1 - Quando um paciente procura informação sobre sua condição de saúde (por exemplo, na internet), isso deve ser considerado pelo médico e discutido, pois demonstra o envolvimento do paciente com seu processo de cuidado

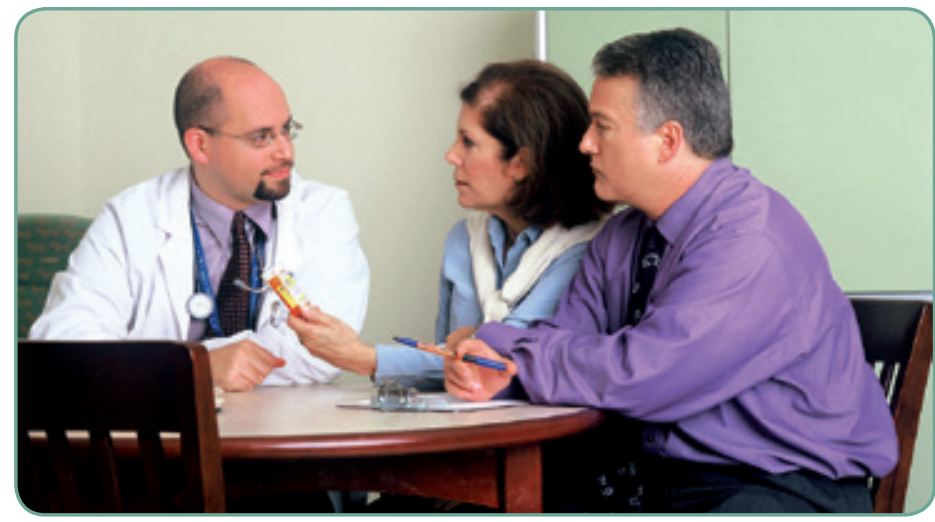

Fonte: Wikimedia Commons (2008).

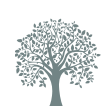

Para mais detalhes sobre factores determinantes para o engajamento, leia: Coulter (2011); Davis et al. (2007) ; Martin, Larsen (2012). 
Ainda no que diz respeito ao paciente, tem sido dada cada vez maior relevância às crenças dos pacientes, em especial sobre a vulnerabilidade para a ocorrência de eventos adversos, competência para o envolvimento e atribuição de papel no processo de saúde e doença. Os resultados dos estudos apontam para níveis mais elevados de motivação para o envolvimento, associados a crenças de maior vulnerabilidade a incidentes e de atribuição de competência para sua prevenção, e atribuição de um papel mais ativo em relação à sua saúde, à doença e ao seu tratamento. A esse respeito, é importante repetir que assistimos, nos nossos dias, ao aumento de vontade expressa dos pacientes em ser considerados como parceiros nesses processos.

\section{Quanto aos factores relativos à doença e às tarefas}

Estudos preliminares associam níveis mais elevados de envolvimento em condições clínicas menos incapacitantes. Esses resultados são entendíveis se pensarmos que pacientes com condições clínicas mais severas ou incapacitantes estão, normalmente, mais debilitados, sentem-se menos conhecedores das questões associadas à gravidade do seu estado, estando, por isso, mais incapazes de tomar a responsabilidade na tomada de decisão. No entanto, em relação a esse aspeto, é de referir a existência de estudos, como o realizado por Stewart et al. (2000), no qual mulheres com cancro/câncer de ovário com pior prognóstico (isto é, maior possibilidade de metastização) mostraram estar mais envolvidas do que as que tinham melhor prognóstico.

Quadro 2 - Determinantes do envolvimento do paciente

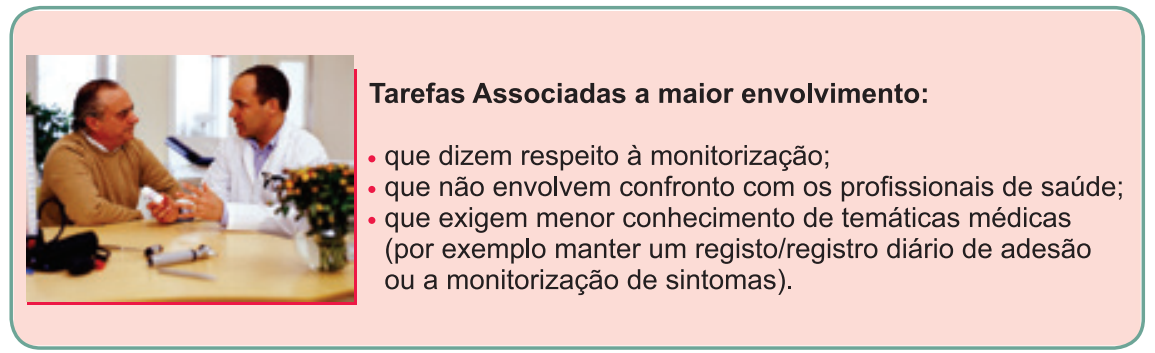

Fonte: Wikipédia (2006)

\section{Quanto aos factores relacionados com os profissionais de saúde}

Parece claro que o envolvimento do paciente quer no que diz respeito à preparação, quer quanto ao nível de envolvimento, quer em relação às áreas ou tarefas de envolvimento e à efetiva importância atribuída a esse envolvimento é determinado pela influência e intervenção do 
profissional de saúde e pela coerência entre as crenças e vontades do paciente e as crenças e vontades do profissional.

A importância que atribuímos a esse factor leva ao seu aprofundamento nos pontos seguintes deste capítulo.

\section{Envolvimento do paciente na segurança dos cuidados de saúde - perspetiva dos profissionais de saúde}

\section{Envolvimento do paciente e o modelo de centração no paciente}

"A era da medicina paternalista, onde o médico conhece o melhor para o paciente, e este se sente afortunado com isso, terminou" (Specter 2013).

Em meados do século passado, o psicanalista Michael Balint, propôs o modelo de centração no paciente precisamente como alternativa aos modelos centrados na doença ou no próprio profissional de saúde (Balint 1969). Esse modelo, que pode ser definido como "os cuidados que respeitam e são responsivos às necessidades, desejos e preferências do paciente e asseguram que todas as decisões são guiadas pelos valores do paciente" (Institute of Medicine 2001, p. 3), é o modelo que sustenta o envolvimento do paciente pelo lado do profissional de saúde. Na verdade, é hoje aceito pela maioria dos autores que afirmam, para evitar os erros provocados pelo modelo paternalista, ser necessário incluir o paciente em todas as fases do processo terapêutico (planeamento/planejamento, tomada de decisão, trabalho em equipa etc.) (Bronkart 2013).

Berwick (2009) destaca três asserções que sintetizam a centração no paciente, a saber:

1. As necessidades do paciente vêm primeiro.

2. "Nada acerca de mim sem mim" e

3. Todo o paciente é único. 
O primeiro ponto (1) enfatiza que o melhor interesse do paciente é o único que deverá ser considerado pelo profissional de saúde. Daqui decorre a necessidade de compreender, com minúcia, a experiência subjetiva do paciente e, quando adequado, da sua família. Efetivamente, o facto do profissional de saúde proceder com a melhor das intenções não garante a satisfação das necessidades do paciente e dos seus familiares (DiGioia et al. 2010).

No segundo ponto (2), o slogan, lançado pelo Ministério da Saúde do Reino Unido e utilizado mais recentemente (2001) pela Fundação Nacional de Segurança dos Pacientes (EUA), "Nada acerca de mim, sem mim" preconiza a necessidade de transparência e participação do paciente e exige que o profissional de saúde se comunique de forma eficaz (Bronkart 2013).

E, por último, o terceiro ponto (3) perspetiva o paciente como único e remete para a necessidade de o profissional dever considerar-se um "convidado" na vida do paciente, bem como para a aceitação da personalização dos cuidados prestados. Seguindo esses três pontos, fica claro que toda a equipa de saúde trabalhará empenhada em colmatar/atenuar as necessidades do paciente e dos seus familiares (DiGioia et al. 2007).

\section{Exigências do modelo de centração no paciente:}

- Partir da situação do paciente (explorar significações de doença e compreender a pessoa em sua totalidade).

- Legitimar a experiência de doença (por exemplo, reconhecer as preocupações, sentimento de perda do paciente).

- Aceitar o paciente como especialista (acreditar na capacidade do paciente para confrontar a situação de doença ou manter a sua saúde).

- Oferecer esperança realista (facultar esperança e suporte no contexto da incerteza ou de prognóstico reservado).

- Desenvolver uma parceria (essa parceria é negociada ao longo do tempo e assenta nas capacidades do paciente).

- Providenciar apoio para o paciente dentro no próprio sistema de saúde (guiar e apoiar o paciente no sistema de saúde).

Centrado no princípio ético da autonomia (Neves 2006), o consentimento informado surge como um excelente exemplo da necessidade de envolvimento do paciente pelo lado do profissional de saúde. Afastando-se inequivocamente da perspetiva de Hipócrates, de quem ficou 
célebre a frase: "Fiz tudo ocultando ao paciente a maior parte das coisas [...]" (Hipocrates - 460-377 a.C. - Sobre a decência), o consentimento informado é hoje um conceito de tendência universal e pressupõe que “o paciente tem o direito a receber, e o médico o dever de prestar o esclarecimento sobre o diagnóstico, a terapêutica e o prognóstico da sua doença" (Ordem dos Médicos, n.1, art. 44, 2008).

O consentimento livre e informado requer que o profissional, após certificação da capacidade de discernimento do paciente, forneça informação de forma clara e honesta a respeito da natureza, duração, objetivos, riscos, efeitos e inconvenientes de um procedimento médico (por exemplo, exame de diagnóstico, tratamento) (Silva 1998). O profissional terá assim de, por meio de uma relação de pareceria (modelo de centração), confirmar a real compreensão do paciente e esclarecer suas eventuais questões, de modo a que esse último possa realizar uma escolha consciente e informada (Muniz, Fortes 1998).

\section{Benefícios e desafios do modelo de centração no paciente para os profissionais de saúde}

Os profissionais de saúde apontam como principais benefícios do envolvimento do paciente a maior participação desse último, a automonitorização da saúde e a melhor compreensão da condição de saúde/doença, bem como dos tratamentos propostos (European Commission 2012). Não obstante a noção clara das vantagens que o envolvimento do paciente aporta, parece existir, ainda, um longo caminho a percorrer no sentido da adoção plena desse modelo. Com efeito, o cidadão comum mantém a perceção que os profissionais de saúde não respondem às questões e preocupações dos pacientes (Entwistle et al. 2005), e, em muitas situações, a afirmação de Slack e colaboradores (1970), “os pacientes são o recurso mais desaproveitado na saúde", continua a ser verdadeira.

\section{Para refletir}

Como profissional da área de saúde, como você vê a questão do envolvimento do paciente? Que benefícios você acredita que, na prática, esse tipo de postura pode trazer? E quais seriam os pontos negativos (caso existam)?

Que dificuldades você já experimentou ou prevê poder vir a experienciar na adoção dessa orientação?

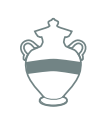

Importa notar que existem limites à aplicação do consentimento livre e informado que não cabem neste capítulo, nomeadamente os casos dos menores ou de adultos incapazes, e as situações de urgência (Oliveira, Pereira 2006).

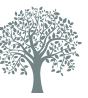

Para saber mais sobre consentimento informado, consulte:

- Muñoz R, Fortes P. O princípio da autonomia e o consentimento livre e esclarecido. In: Conselho Federal de Medicina. Iniciação à bioética. Brasília, DF: CFM; 1998. p. 53-70.

- Conselho Federal de Medicina. Código de médica ética. Brasília, DF: CFM; 2009. artigos 46, 48, 56, 59, 123 e 124.

- Oliveira G. Pereira, A. Consentimento informado. Coimbra: Centro de Direito Biomédico, 2006. 
Na realidade, durante muito tempo, acreditou-se que os pacientes não poderiam contribuir com nada de interessante para seu processo terapêutico, uma vez que a saúde/doença engloba áreas muito específicas sobre as quais apenas profissionais altamente qualificados detinham conhecimento (Bronkart 2013). Não admira, portanto, que a literatura atual destaque algumas dificuldades que emergem quando os profissionais procuram utilizar um modelo mais centrado no paciente. Alguns manifestam certa apreensão perante a possibilidade de que, ao envolver o paciente, ele possa, por um lado, discordar abertamente do profissional de saúde e, por outro lado, tente diagnosticar-se e tratar-se a si próprio (European Commission 2012) fazendo escolhas "erradas". Sem negar a possibilidade que tal aconteça, Berwick (2009) salienta que essas situações constituem exceções, e casos extraordinários não devem servir de base para a construção de regras. Outra dificuldade advém da sensação de que, ao trabalhar de acordo com o modelo de centração no paciente, o clínico negligencia os seus próprios desejos e necessidades. Ora, como é sabido, esse modelo pressupõe que o profissional tome consciência das suas emoções e retome os princípios básicos de ajuda que caracterizam a relação profissional de saúde-paciente.

Por último, e com maior ênfase na literatura, surge a crença de que o envolvimento do paciente acarreta necessariamente mais custos. Contrapondo essa perspetiva, diversos estudos recentes (por exemplo, DiGioia et al. 2010) têm vindo a demonstrar que a centração no paciente melhora os resultados de saúde sem acréscimo financeiro.

\section{Limites dos profissionais de saúde e necessidade de envolvimento do paciente}

Os profissionais de saúde não podem responder às necessidades dos pacientes se eles não as expressam; para além disso, não conseguem diagnosticar com rigor ou prescrever com segurança se a informação relevante não consta do registo médico ou está incorreta. Nesse contexto, existem, atualmente, autores (por exemplo, Bronkart 2013; Coulter 2012), que poderemos apelidar de mais "radicais", defensores do livre acesso ao registo médico pelos pacientes e suas famílias, argumentando que se trata de uma forma especialmente eficaz de detetar erros e, como consequência, evitar incidentes. Os mesmos autores enfatizam que, considerando o constante progresso da medicina, é hoje humanamente impossível para qualquer profissional de saúde ser detentor de todo o conhecimento científico existente na sua área do saber. A título de exemplo, só em 2010, foram indexados ao sistema de publicação da Medline 800 mil artigos. Dessa forma, quando um paciente inicia um processo de procura de informação, especialmente 
em situações de pior prognóstico ou mais raras (por exemplo, alguns tipos de cancro/câncer), ele não deverá ser encarado com desconfiança, mas sim como possível coadjuvação ao próprio profissional.

São, aliás, cada vez mais comuns as situações em que o paciente confronta o clínico com possibilidades de tratamento encontradas por meio de pesquisas pessoais. Dave de Bronkart é atualmente o exemplo mais divulgado dessas situações. Após o diagnóstico de cancro/câncer raro em fase terminal, Bronkart encontrou, fazendo suas pesquisas, um tratamento desconhecido pelo seu médico e que lhe salvou a vida. Essa experiência levou o paciente a tornar pública sua condição e iniciar uma cruzada no sentido de maior envolvimento do paciente nos cuidados de saúde.

Longe de considerar que tudo o que os pacientes encontram, nomeadamente na internet, aporta bons resultados terapêuticos, os autores parecem unânimes quanto à necessidade de valorizar a informação facultada no contexto da relação médico-paciente e encorajar o envolvimento do paciente (veja como no destaque a seguir) (Bronkart 2013; Coulter 2012).

\section{Dez verbalizações que os profissionais podem utilizar para} encorajar o envolvimento do paciente

1. "Estou aqui para trabalhar em conjunto consigo em prol da sua saúde. Somos uma equipa."

2. "Aprenda o mais que puder acerca da sua condição. Aqui tem algumas formas possíveis por onde pode começar..."

3. "Fale sobre a sua condição com outros pacientes em situações idênticas."

4. "Eu encorajo-o a pedir uma segunda opinião antes de decidir em relação a uma grande cirurgia ou outros tratamentos igualmente sérios."

5. "Eu não tenho a resposta para a sua questão; vamos procurar os dois juntos."

6. "Existe um número considerável de opções, cada uma com prós e contras. Vamos falar daquela que prefere."

7. "Aqui tem a forma como pode contatar-me via electrónica/email."

8. "Estes são os assuntos que eu gostaria de abordar consigo hoje: Quais sãos as suas preocupações?"

9. "Consegui abordar todas as suas preocupações? Há mais alguma coisa?"

10. "Sinta-se à vontade para ler os seus resultados dos exames e o resto do seu registo médico disponível on-line sempre que desejar. Também poderá utilizar o website para agendar um encontro, solicitar uma requisição ou realizar outras tarefas administrativas."

Fonte: Bronkart (2013, p. 49)

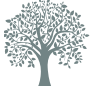

Para saber mais sobre esse caso, visite o endereço: http:// www.ted.com/talks/dave_ debronkart_meet_e_patient_ dave.html 


\section{Envolvimento do paciente na segurança dos cuidados de saúde - perspetiva das organizações ou dos serviços}

\section{Cultura de segurança nas organizações: o envolvimento do paciente como prioridade}

Para que o envolvimento do paciente seja concretizado de forma consistente, torna-se imperativo que os serviços de saúde coloquem os pacientes no topo das suas prioridades (DiGioia et al. 2007). O mesmo é dizer que o envolvimento do paciente e da família, assim como sua integração na forma de parceiros efetivos da equipa de saúde deverão constituir-se como um dos principais objetivos de qualquer serviço de saúde (European Commission 2012). Os pacientes e família precisam ser tratados com dignidade e respeito por todos os membros da organização e encorajados a reportar preocupações relacionadas com a segurança. Na verdade, a probabilidade de os pacientes aderirem aos tratamentos e, consequentemente, alcançarem melhores resultados é maior quando eles sentem suporte não apenas por parte dos clínicos, mas também de toda a organização de saúde que lhes presta cuidados (Joint Commission Resources 2006).

Líderes e membros da organização de saúde necessitam criar uma cultura de segurança que se traduz pela plena adoção do modelo de centração no paciente (Joint Commission Resources 2006), em oposição ao modelo tradicional. Nesse modelo, o foco da organização era colocado no estabelecimento de rotinas que funcionam para os profissionais de saúde, mas que não promovem a qualidade e contribuem para a insatisfação dos pacientes e das suas famílias, e os pacientes, mais que ajudados, podem ser lesados (Kohn et al. 1999).

Essa perspetiva requer a reestruturação de muitos serviços, sendo essencial a existência de lideranças concentradas em remover barreiras (DiGioia et al. 2007), promover a mudança de atitudes por parte de todos os membros da organização (Joint Commission Resources 2006) e facilitar as práticas necessárias para alcançar os objetivos (DiGioia et al. 2007). Trata-se de um processo que requer tempo, pois depende do estabelecimento de relações baseadas na comunicação aberta e na confiança mútua entre líderes, administradores, restantes membros da organização e pacientes. Não admira, portanto, que essa mudança origine certo grau de desconforto no seio de algumas organizações. Contudo, esse parece ser um caminho sem retrocesso possível, pois a realidade atual demonstra, de forma clara, que, por um lado, quando 
a segurança é baseada por uma cultura da culpa, ela gera animosidades e relações tensas entre os elementos da equipa. Por outro lado, a incapacidade para se comunicar de forma aberta e respeitosa com os pacientes contribui para a ocorrência de erros médicos, que podem provocar danos ou mesmo a morte do paciente.

\section{A criação de serviços que promovem envolvimento do paciente nas organizações}

A ouvidoria em saúde é um instrumento da gestão pública e do controlo social para o aperfeiçoamento da qualidade e da eficácia das ações e serviços prestados [...]. (Ministério da Saúde 2005 p. 13).

Figura 2 - Campanha de divulgação da Ouvidoria do SUS no Brasil

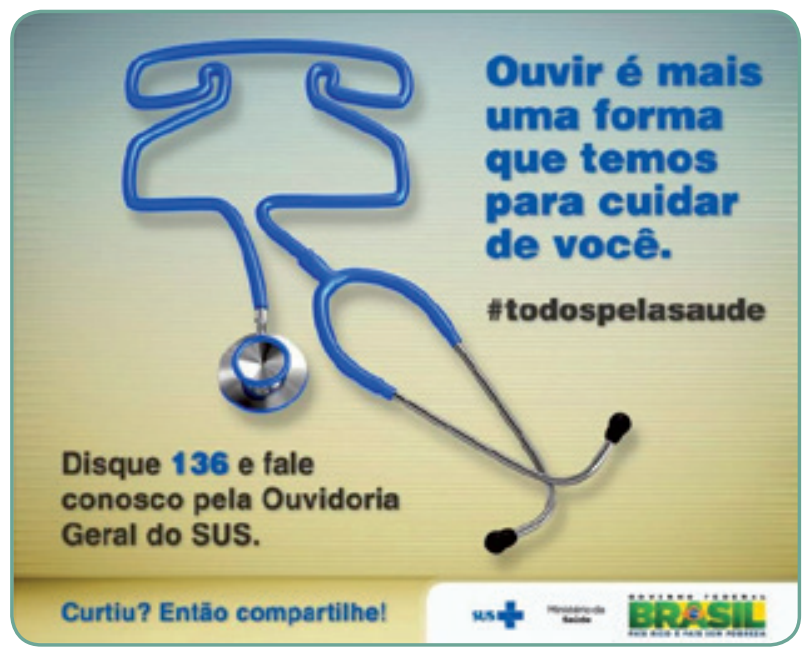

Fonte: Ministério da Saúde do Brasil.

Em algumas organizações que estabeleceram uma clara cultura de segurança, o envolvimento do paciente tem vindo a consubstanciar-se na criação de serviços de ouvidoria de apoio do utente/usuário e familiares. A Joint Commission Resources (2006) salienta que a criação desses serviços terá de ser suportada, em primeira instância, pelos líderes e apenas resultará se a organização de saúde possuir equipas multidisciplinares que trabalhem de forma verdadeiramente eficaz. Essa equipa deverá ser composta de pessoas que demonstrem respeito e sensibilidade para ouvir e assimilar as informações, sejam capazes de atuar com consciência crítica e possuam os conhecimentos necessários para proceder ao melhor encaminhamento de cada caso apresentado pelo paciente (Ministério da Saúde 2005). 


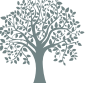

- Para saber mais sobre o caso das pacientes de Boston, consulte: http://www.danafarber.org/Adult-Care/NewPatient-Guide/Adult-Patientand-Family-Advisory-Council. aspx

- O Institute for Family Centered Care disponibiliza informação e materiais para ajudar os interessados em criar e manter gabinetes de ouvidoria para pacientes e familiares no endereço: www. familycenteredcare.org
Esses serviços influenciam positivamente as atitudes dos membros da organização, no sentido de eles envolverem de facto o paciente e sua família como parceiros na prestação de cuidados de saúde. A experiência das instituições que construíram esses gabinetes demonstra que o mais relevante resulta da criação de mecanismos que possibilitem aos pacientes e famílias trabalharem em colaboração com os profissionais de saúde e os administrativos para a contínua promoção dos cuidados prestados, assim como para a educação dos elementos da equipa. A título de exemplo, em 1998, na sequência dos efeitos provocados pelo facto de duas pacientes receberem quatro vezes a sua dose diária de quimioterapia para tratamento de carcinoma mamário, o Instituto do Cancro/Câncer Dana-Fraber, em Boston, empenhou-se em promover ativamente a participação do paciente em todos os aspetos do funcionamento do hospital. Entre outras vertentes, os elementos que fazem parte do gabinete de ouvidoria dessa organização acompanham os médicos nas visitas médicas e são encorajados a perguntar ao paciente como tem sido a experiência do internamento/internação. Essa participação do paciente e da família como parceiros promoveu a qualidade dos cuidados, aumentou a autoestima dos pacientes e incrementou a segurança do paciente ao tornar possível não apenas a identificação atempada/oportuna de potenciais problemas, mas também o desenvolvimento de soluções mais eficazes (isto é, que atendam às necessidades dos pacientes) para os problemas encontrados.

Para refletir

Qual a postura da sua organização em relação aos canais de comunicação com os pacientes? Existe uma ouvidoria ou gabinete do utente/usuário ou serviço ou departamento semelhante? Como você os avalia?

\section{A comunicação dos incidentes ocorridos aos pacientes e famílias (disclosure)}

Num sistema de saúde que se pretende centrado no paciente, a informação sobre a ocorrência de um incidente relacionado com os cuidados de saúde é um imperativo. A informação ao paciente sobre um incidente pode ser definida como a "comunicação entre o profissional de saúde e o paciente ou familiar na qual é reconhecida a ocorrência de um erro, discutido o acontecimento e estabelecida a ligação entre o erro e o seu efeito, de forma que seja percetível para o paciente" (Lazare 2006). Sorensen e colaboradores (2008) referem ainda que "a comunicação aberta é de facto a formalização do processo entre profis- 
sionais de saúde e paciente, e deveria ser a base de toda a comunicação, independentemente de se referir ou não a um incidente".

A maioria dos autores considera essa informação como uma "boa prática", e, em vários questionários a profissionais de saúde, a porcentagem daqueles que têm intenção de comunicar um incidente é elevada, contrastando com o pequeno número daqueles que têm experiência real de o fazer (Sorensen et al. 2008; Gallagher et al. 2006; McIntyre, Popper 1983).

Os pacientes que foram vítimas de um incidente e seus familiares esperam uma informação explícita de como o incidente ocorreu, como será prevenido no futuro e um pedido de desculpas. Para a grande maioria dos pacientes, as medidas que vão tomar após um incidente (decisão positiva ou negativa de litígio) estão diretamente relacionadas com a forma como a informação lhes foi transmitida nos dias imediatos à sua ocorrência (Lazare 2006; Sorense et al. 2008; Boyle et al. 2006; Berwick 2009).

Algumas das barreiras e vantagens sentidas pelos profissionais e pacientes na revelação de eventos adversos e referidas na literatura (Lazare 2006; Boyle et al. 2006; Gallagher et al. 2006; Harvard 2006; Lamb et al. 2003) estão resumidas no Quadro 3.

Quadro 3 - Benefícios e riscos expressos ao revelar incidentes adversos

\begin{tabular}{|c|c|c|}
\hline & Barreiras e riscos & Benefícios \\
\hline $\begin{array}{l}\text { Profissionais } \\
\text { de saúde }\end{array}$ & $\begin{array}{l}\text { Medo de litígio } \\
\text { Falta de coragem } \\
\text { Incerteza sobre conteúdo da entrevista } \\
\text { Falta de treino } \\
\text { Medo de preocupar o paciente } \\
\text { Medo do que o paciente possa dizer } \\
\text { Perda de prestígio/status } \\
\text { Isolamento, falta de apoio } \\
\text { Pressão silenciadora da organização }\end{array}$ & $\begin{array}{l}\text { Alívio } \\
\text { Retomada da relação e da confiança } \\
\text { com o paciente } \\
\text { Feedback do paciente sobre os cuidados } \\
\text { Diminuição da possibilidade de lesão } \\
\text { para outros } \\
\text { Transparência } \\
\text { Deteção de falhas no sistema }\end{array}$ \\
\hline Pacientes & $\begin{array}{l}\text { Medo de retribuição negativa dos } \\
\text { profissionais e da organização } \\
\text { Sentimento de culpa dos familiares } \\
\text { Canais de comunicação difíceis } \\
\text { Medo de ser abandonado nos cuidados } \\
\text { Difícil perceção do erro } \\
\text { Medo de ofender os clínicos } \\
\text { Culpa diluída no sistema }\end{array}$ & $\begin{array}{l}\text { Tratamento adicional } \\
\text { Compensação financeira } \\
\text { Menor ansiedade relativa a sintomas } \\
\text { inexplicados } \\
\text { Maior confiança nos profissionais } \\
\text { Perceção de ser respeitado por parte } \\
\text { dos profissionais } \\
\text { Contribuição para a prevenção e análise } \\
\text { do incidente }\end{array}$ \\
\hline
\end{tabular}




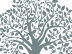

(3)

Para aprofundar esse tema, veja mais informações sobre o protocolo SPIKES Protocolo de seis passos para comunicar más notícias (Baile W, et al. SPIKES: a six-step protocol for delivering bad news. Oncologist. 2000 Aug; 5(4):302-11).
O Consensus Statement of the Harvard Hospitals: Responding to Adverse Events, publicado em março de 2006 (Harvard 2006), é um excelente guia para todo o processo de informação ao paciente relativo a um evento adverso, percorrendo, de forma prática e objetiva, as questões fundamentais de quais são os incidentes a serem revelados, por quem, quando e como. Sugere a necessidade de apoio para a vítima do incidente (cuidados de saúde gratuitos), mas também de uma estrutura dinâmica de apoio aos profissionais envolvidos ("emotional first aid"). Várias organizações de saúde nas quais uma política de comunicação aberta é incentivada verificaram, paradoxalmente, diminuição do número de reclamações e processos litigiosos (Lamb et al. 2003; Berwick 2003; Berwick 2009).

\section{Estratégias para a promoção do envolvimento do paciente}

Depois de abordar as questões principais do envolvimento do paciente em matérias de segurança, cabe, agora, sistematizar algumas estratégias de promoção do envolvimento (Quadro 4) não apenas para o paciente, mas também para os profissionais de saúde e para as próprias organizações de saúde. Várias dessas estratégias requerem mudanças de atitudes e até treino de competências.

Quadro 4 - Estratégias e promoção do envolvimento do paciente

\begin{tabular}{|c|c|c|}
\hline Paciente & Profissionais de saúde & Organizações de saúde \\
\hline $\begin{array}{l}\text { Desenvolver o sentido positivo } \\
\text { do envolvimento }{ }^{1} \\
\text { Promoção da literacia }{ }^{2} \\
\text { Treino das competências } \\
\text { comunicacionais }{ }^{2}\end{array}$ & $\begin{array}{l}\text { Motivar e dotar de } \\
\text { competências para a utilização } \\
\text { do modelo de centração no } \\
\text { paciente }^{1} \\
\text { Promoção de atitudes } \\
\text { facilitadoras do envolvimento } \\
\text { do paciente }{ }^{1} \\
\text { Treino das competências } \\
\text { comunicacionais }{ }^{1} \\
\text { Utilizar o consentimento } \\
\text { livre e informado de forma } \\
\text { apropriada } \\
\text { Comunicações de incidentes } \\
\text { aos pacientes }\end{array}$ & $\begin{array}{l}\text { Cultura de segurança do } \\
\text { paciente } \\
\text { Formação das lideranças para } \\
\text { o envolvimento do paciente } \\
\text { Treino do trabalho em equipa } \\
\text { multidisciplinar } \\
\text { Criação de gabinetes do } \\
\text { utente/usuário/ouvidoria, } \\
\text { envolvendo os pacientes como } \\
\text { parceiros integrais em todos os } \\
\text { aspetos da organização² }\end{array}$ \\
\hline
\end{tabular}

${ }^{1}$ Entwistle, Watt (2006)

2 Joint Commission Resource (2006) 


\section{Considerações finais}

Ainda que se mostre desejável, a promoção do envolvimento do paciente e da família na sua segurança deverá ser orientada pelo conhecimento claro não só do âmbito e das oportunidades para esse envolvimento, mas igualmente dos seus limites e obstáculos. No entanto, o envolvimento efetivo do paciente implica sempre que ele tenha oportunidade para avaliar e discutir os cuidados que recebe, com a certeza de que os profissionais que prestam esses cuidados utilizarão essa informação de forma construtiva, orientada para a melhoria da sua qualidade.

Por último, importa ressaltar que, nas próximas décadas, as escolas de saúde serão chamadas a alertar os profissionais de saúde para a importância da comunicação eficaz com os pacientes, não apenas porque possibilita maior satisfação (Cvengros et al. 2007), aumento da adesão ao tratamento (Arbuthnott, Sharpe 2009; DiMatteo 2004), restabelecimento mais rápido do paciente (Street 2009) e menor perturbação emocional (Corney 2000), mas, principalmente, porque se trata de uma importante ferramenta para a promoção da segurança do paciente e prevenção de incidentes (Gong et al. 2006). Na maioria das escolas, esse desafio acarreta alterações substanciais na sua oferta formativa, uma vez que os resultados de estudos realizados com estudantes e profissionais apontam, de forma inequívoca, para a necessidade da promoção do treino de competências comunicacionais no ensino pré e pós-graduado (Grilo 2010). Assim, é necessário que o ensino das competências comunicacionais no ensino pré-graduado se prolongue até a entrada na vida profissional. Deverá tratar-se de um processo contínuo, com início nos primeiros anos da formação dos futuros profissionais, e vá acompanhando os contactos desses com os pacientes em contexto de supervisão (Grilo 2010; Joint Commission Resources 2006). Já no decurso da vida profissional, importa considerar que os profissionais necessitam de tempo e esforço para corrigir suas falhas (Fallowfield et al. 1998), justificando-se a inclusão de programas de treino que partam das competências comunicacionais de cada profissional, de forma a responder mais adequadamente às suas necessidades específicas (Rollnick, Kinnersley \& Butler 2002).

\section{Referências}

Adams RJ, Smith BJ, Ruffin RE. Patient preferences for autonomy in decision making in asthma management. Thorax. 2001 Feb;56(2):126-32.

Agência Nacional de Vigilância Sanitária. 2012. Disponível em: www.anvisa.gov.br. 
Agency of Healthcare Research and Quality. Five steps to safer healthcare: patient fact sheet. Rockville, MD: AHRQ; 2004.

Arbuthnott A, Sharpe D. The effects of physician-patient collaboration on patient adherence nonpsychiatric medicine. Patient Educ Couns. 2009;77(1):60-7.

Balint M, Ball DH, Hare ML. Training medical students in patient-centered medicine. J Compr Psychiatry. 1969;10(4):249-58.

Berwick D. Errors today and errors tomorrow. N Engl J Med. 2003;348;25-19.

Berwick, D. What "patient-centered" should mean: confessions of an extremist. Health Aff. 2009;28(4): w555-5.

Boyle D, O'Connell D, Platt FW, Albert RK. Disclosing errors and adverse events in the intensive care unit. Crit Care Med. 2006;34:1532-37.

Bronkart D. "Let patients help": a patient engagement handbook. USA: Create Space Independent Publishing Platform; 2013.

Center for Advancing Health. A new definition of patient engagement: hat is engagement and why is it important? Washington, D: CAH; 2010.

Connock M, Stevens C, Fry-Smith A, Jowett S, Fitzmaurice D, Moore D, Song F. Clinical effectiveness and cost-effectiveness of different models of managing long-term oral anticoagulation therapy: a systematic review and economic modelling. Health Technol Assess. 2007;11(38):iii-iv, ix-66.

Conselho Federal de Medicina. (2009) Código de médica ética. Brasília, DF: CFM; 2009.

Corney R. O desenvolvimento das perícias de comunicação e aconselhamento na medicina. Lisboa: Climepsi Editores; 2000.

Coulter A. Engaging patients in healthcare. New York: Open University Press; 2011.

Coulter A. Engaging patients in their healthcare. Oxford: Picker Institute Europe; 2006.

Coulter A, Parsons S, Askham J. Where are the patients in in decision-making about their own care? Geneva: World Health Organization; 2008.

Cvengros J, Christensen A, Hillis S, Rosenthal G. Patient and physician attitudes in the health care context: attitudinal symmetry predicts patient satisfaction an adherence. Ann Behav Med. 2007;33(3):262-8

Davis RE, Jacklin R, Sevdalis N, Vincent CA. Patient involvement in patient safety: what factors influence patient participation and engagement? Health Expect. 2007 Sep;10(3):259-67. 
Dentzer S. Rx for the 'blockbuster drug' of patient engagement. Health Aff. 2013;32(2):202.

DiGioia A, Greenhounse P, Levison, T. Patient and Family-centered collaborative care. Clin Orthop Relat Res. 2007;463:13-9.

DiGioia, A, Lorenz H, Greenhouse P, Bertoty D, Rocks, S. A patient-centered model to improve metrics without cost increase. J Nurs Adm. 2010 Dec;40(12): 540-6.

DiMatteo MR. The role of effective communication with children and their families in fostering adherence to pediatric regimens. Patient Educ Couns. 2004;55(3):339-44.

Douketis JD. Patient self-monitoring of oral anticoagulant therapy: potential benefits and implications for clinical practice. Am J Cardiovasc Drugs. 2001;1(4):245-51.

Entwistle VA, Mello MM, Brennan TA. Advising patients about patient safety: current initiatives risk shifting responsibility. Jt Comm J Qual Patient Saf. 2005 Sep;31(9):483-94.

Entwistle VA, Watt I. Patient involvement in treatment decision-making: the case for a broader conceptual framework. Patient Educ Couns. 2006;63(3):268-78.

European Commission. Eurobarometer qualitative study: patient involvement. Brussels: DirectorateGeneral for Health and Consumers; 2012.

Fallowfield L, Lipkin M, Hall A. Teaching senior oncologists communication skills: results from phase I of a comprehensive longitudinal program in the United Kingdom. J Clin Oncol. 1998;16(5): 1961-68.

Finkelstein J, Knight A, Marinopoulos S, et al. Enabling patient-centered care through health information technology. Rockville, MD: Agency for Healthcare Research and Quality; 2012 Jun. (AHRQ Publication, No. 12-E005-EF).

Gallagher TH, Garbutt JM, Waterman AD, et al. Choosing your words carefully. How physicians would disclose harmful medical errors to patients. Arch Intern Med. 2006;166:1585-93.

Gong Y, Zhu M, Li J, Turley J, Zhang J. Clinical communication ontology for medical errors. AMIA Annu Symp Proc. 2006:930.

Grilo AM. Processos comunicacionais em estudantes de fisioterapia e fisioterapeutas: categorização e proposta de um treino individual de competências [tese]. Lisboa: Faculdade de Psicologia, Universidade de Lisboa; 2010.

Hall J, Peat M, Birks Y, Golder U, et al. Effectiveness of interventions designed to promote patient involvement to enhance safety: a systematic review. Qual Saf Health Care. 2010 Oct;19(5):e10. doi:10.1136/qshc.2009.032748.

Harvard Hospitals. When things go wrong: responding to adverse events: a consensus Statement. [Local desconhecido]: Harvard Hospitals; 2006. 
Hibbard J, Green J. What the evidence shows about patient activation: better health outcomes and care experiences; fewer data on costs. Health Aff. 2013;32:2207-14. doi:10.1377/ hlthaff.2012.1061

Hudon C, Fortin M, Haggerty J, Loignon C, Lambert M, Poitras M. Patient-centered care in chronic disease management: a thematic analysis of the literature in family medicine. Patient Educ Couns. 2012;88:170-6.

Institute of Medicine. Crossing the quality chasm: a new health system of the 21 st century. Washington, DC: National Academies Press, 2001.

Joint Commission. Patient as partners: how to involve patients and families in their own care. Oakbrook Terrace, IL: Joint Commission on Accreditation of Healthcare Organizations; 2006.

Joint Commission. Speak up initiatives. Oakbrook Terrace, IL: Joint Commission; 2004.

Joint Commission on Accreditation of Healthcare Organization. Joint Commission Resources Mission: patients as partners. Oakbrook Terrace, IL: Joimt Comission; 2006 [citado 2014 Set 5]. Disponível em: http://www.jcrinc.com.

Joint Commission on Accreditation of Healthcare Organization. Speak up: help prevent errors in your care. Oakbrook Terrace, IL: Joimt Comission; 2005 [citado 2014 Set 5]. Disponível em: http:// www.jcahao.org/accredited+organizations/speak+up/speak+up+index.htm.

Joint Commission Resources. Case study: Ingham Regional Medical Center involves patients in the infection control process. Jt Comm Perspect Patient Saf. 2005 Apr; 5:9-10.

Joyce-Moniz L, Barros L. Psicologia da doença para cuidados de saúde: desenvolvimento e intervenção. Lisboa: Edições Asa; 2005.

Kohn LT, Corrigan JM, Donaldson MS, editors. To err is human: building a safer health system. Washington, DC: National Academy Press, Institute of Medicine; 1999.

Koutantji M, Davis R, Vincent C, Coulter. The patient's role in patient safety: engaging patients, their representatives, and health professionals. Clin Risk. 2005;1199-104.104.

Lamb RM, Studdert DM, Bohmer RMJ, Berwick D, Brennan TA. Hospital disclosure practices: results of a national survey. Health Aff. 2003;22(2):73-83.

Lazare A. Apology in medical practice: an emerging clinical skill. JAMA. 2006;296:1401-04.

Martin $\mathrm{H}$, Larsen J. Patient involvement in patient safety: a literature review about European primary care. Copenhagem: The Danish Institute for Health Services Research; 2012.

Mclntyre N. Popper K. The critical attitude in medicine: the need for a new ethics. BMJ. 1983;287:1919-23.

Ministério da Saúde (Brasil). Política Nacional de Atenção Básica. Brasília, DF: MS; 2007. 
Ministério da Saúde (Brasil), Secretaria de Gestão Participativa. Oficinas de Ouvidoria. Brasília, DF: Departamento de Ouvidoria-Geral do SUS; 2005.

Muñoz R, Fortes P. O princípio da autonomia e o consentimento livre e esclarecido. In: Conselho Federal de Medicina. Iniciação à bioética. Brasília, DF: CFM; 1998. p. 53-70.

National Health Services (NHS). Plan for investment; plan for reform. London: Secretary of State for Health By Command of Her Majesty UK; 2000.

Neves N. Ética para futuros médicos: é possível ensinar? Brasília, DF: Conselho Federal de Medicina; 2006.

Oliveira G. Pereira, A. Consentimento informado. Coimbra: Centro de Direito Biomédico, 2006.

Ordem dos Médicos (Portugal). Código deontológico. Lisboa: OM; 2008.

Osler W. A way of life. New York: Harper \& Row, 1937

Piker Institute Europe, Department of Health. Invest in engagement. Oxford: Picker Institute Europe; 2010.

Rao J, Weinberger M, Kroenke K. Visit-s+ecific expectations and patient-centered outcomes: a literature rewie. Arch Family Med. 2000;9:1148-55.

Rollnick S, Kinnersley P, Butler C. Context-bound communication skills training: development of a new method. Med Educ. 2002;36:377-83.

Santos M, Grilo A, Andrade G, Guimarães T. Comunicação em saúde e segurança do paciente: problemas e desafios. Rev Port Saúde Pública. 2010;10:47-57.

Secretary of State for Health England. The national health plan: a plan for investment, a plan for reform. London: The Stationery Office; 2002.

Silva F. Da ética filosófica à ética em saúde. In: Conselho Federal de Medicina. Iniciação à bioética. Brasília, DF: CFM; 1998. p. 19-38

Slack WV, Van Cura LJ, Greist JH. Computers and doctors: use and consequences. Comput Biomed Res. 1970 Oct 5; 3(5):521-7.

Sorensen R, ledema R, Piper D, et al. Health care professionals views of implementing a policy of open disclosure of errors. J Health Serv Res Policy. 2008;13:227-32.

Specter M. The New Yorker, 2013 Feb 4.

Stewart DE, Wong F, Cheung AM, Dancey J, Meana M, Cameron Jl et al. Information needs and decisional preferences among women with ovarian cancer. Gynecol Oncol. 2000;77:357-61. 
Street RL, Makoul G, Arora NK, Epstein RM. How does communication heal? Pathways linking clinician-patient communication to health outcomes. Patient Educ Couns. 2009;74:295-301.

Studdert DM, Mello MM, Gawande AA, et al. Claims, errors, and compensation payments in medical malpractice litigation. N Engl J Med. 2006;354(19):2024-33.

TNS Qual+; European Commission. Eurobarometer qualitative study: patient involvement: aggregate report 2012. Brussel: TNS Qual+, 2012.

UK. Department of Health. Equality and excellence: liberating de NHS. London: The Stationery Office, 2010 Jul.

Vincent CA, Coulter A. Patient safety: what about the patient? Qual Safe Health Care. 2002;11(1):76-80.

Vincent $C$. et al The patient's role in patient safety: engaging patients, their representatives, and health professionals. Clin Risk 2005;1199-104.104.

Waterman AD, Gallagher TH, Garbutt J, Waterman BM, Fraser V, Burroughs TE. Hospitalized patients' attitudes about and participation in error prevention. J Gen Intern Med. 2006;21:367-70.

Weissman JS, Schneider EC, Weingart SN, Epstein AM, David-Kasdan J, et al. Comparing patientreported hospital adverse events with medical record review: do patients know something that hospitals do not? Ann Intern Med. 2008 Jul 15;149(2):100-8.

Weingart SN, Zhu J, Chiappetta L, et al. Hospitalized patients' participation and its impact on quality of care and patient safety. Int J Qual Health Care. 2011;23:269-277L.

Wikimedia Commons. File:Doctor and couple talking (1).jpg. 2008 [citado 2014 Out 21]. Disponível em: http://commons.wikimedia.org/wiki/File:Doctor_and_couple_talking_(1).jpg

Wikipédia. Relación médico paciente. 2006 [citado 2014 Out 21]. Disponível em: http://en.wikipedia. org/wiki/Medicalization\#mediaviewer/File:Relaci\%C3\%B3n_M\%C3\%A9dico_Paciente.png

World Health Organization (WHO). Adherence to long-term therapies: evidence for action. Geneva: WHO; 2008 [citado 2013 Jan 26]. Disponível em: ww.who.int/chronic_conditions/adherencereport/en.

World Health Organization (WHO). Declaration of Ata Alma. Geneva: WHO; 1978.

World Health Organization (WHO) .Patient Safety Program: patient for patient safety. Geneva: WHO; 2006.

Zwaan L, Bruijne M, Wagner C, Thijs A, Smits A, et al. Patient record: review of the incidence consequences and causes of diagnostic adverse events. Arch Int Med. 2010;170(12):28. 